\title{
Dysregulation of Toll-Like Receptors in Dogs with Chronic Enteropathies
}

Schnyder $\mathbf{M}^{1}$, Oevermann $\mathbf{A}^{1}$, Doherr $\mathbf{M G}^{1}$, Luckschander $\mathbf{N}^{2}$, Zurbriggen $\mathrm{A}^{1}$ and Burgener $\mathbf{I A}^{2^{*}}$

${ }^{1}$ Department of Clinical Veterinary Medicine (Schnyder) and the Department of Clinical Research and Veterinary Public Health, Vetsuisse Faculty, University of Bern, Switzerland

2Department for Small Animals and Horses, University of Veterinary Medicine, Vienna, Austria

*Corresponding author: Burgener IA, Department for Small Animals and Horses, University of Veterinary Medicine, Vienna, Austria, E-mail: iwan.burgener@vetmeduni.ac.at

Received date: August 03, 2018; Accepted date: September 10, 2018; Published date: September 19, 2018

Copyright: (c) 2018 Schnyder M, et al. This is an open-access article distributed under the terms of the Creative Commons Attribution License, which permits unrestricted use, distribution, and reproduction in any medium, provided the original author and source are credited.

\section{Abstract}

Background: Toll-like receptors (TLRs) recognize microbe-associated molecular patterns (MAMPs) and play an important role in the regulation of inflammation in the gastrointestinal tract.

Hypothesis/objectives: There is a dysregulation of TLRs recognizing bacterial MAMPs at the protein level in canine chronic enteropathies (CCE).

Animals: 20 healthy control dogs (HCD), 20 dogs with steroid-responsive (SR) and 20 dogs with food-responsive (FR) diarrhea.

Methods: Prospective study. Biopsies from duodenum and colon were taken before and after standard therapy in 40 dogs with SR and FR and at necropsy in 20 healthy beagles. Immunohistochemistry was performed to determine the expression of TLR2, 4, 5 and 9 using immunohistochemistry.

Results: TLR2 positive cells were downregulated in the duodenum and colon in FR and SR compared to HCD before and after therapy (all $p<0.0001$ ). In regard to the epithelium, the expression of TLR2 was higher in FR before therapy $(p=0.009)$ compared to HCD. TLR4 positive cells were upregulated in duodenum in FR before ( $p=0.008)$ and after $(p=0.02)$ therapy compared to HCD. In the colon, TLR4 positive cells were significantly upregulated in FR and SR before $(p=0.0009)$ and after $(p=0.0002)$ therapy in comparison to HCD. No expression of TLR4 was seen in the epithelium. TLR5 positive cells were upregulated in the colon in SR and FR compared to HCD before therapy $(p=0.005)$ and in FR after therapy $(p=0.020)$. TLR9 positive cells were significantly decreased in the duodenum and colon of dogs with SR and FR before TLR5 positive cells were upregulated in the colon in SR and FR compared to HCD before therapy $(p=0.005)$ and in FR after therapy $(p=0.020)$ and after therapy compared to HCD $($ all $p<0.0001)$. In regard to the epithelium, the expression of TLR9 was significantly lower in FR before and in FR and SR group after therapy in duodenum and colon compared to HCD.

Conclusion and clinical importance: In this study, TLR4 and TLR5 expression at the protein level was significantly upregulated in inflammatory cells in CCE leading to increased inflammation. The downregulation of TLR9 in cells and epithelium seen in CCE compared to healthy dogs points towards the important role that TLR9 also plays in gut homeostasis in mice and men. Further studies are needed to evaluate the potential use of new therapeutic approaches to impair TLR4 and 5 expression or induce TLR9 activation with corresponding ligands (e.g., synthetic lipopeptides or synthetic CpGs, respectively) in CCE.

Keywords Inflammatory bowel disease; Food-responsive diarrhea; Commensal bacteria; Diarrhea

\section{Introduction}

Canine chronic enteropathies (CCE) are a common cause for vomiting and diarrhea in dogs and include inflammatory bowel disease (IBD; sometimes also called steroid-responsive diarrhea (SR)) and food-responsive diarrhea (FR). IBD is characterized by persistent or recurrent clinical signs of gastrointestinal disease of unknown cause associated with histologic evidence of inflammation in the small and/or large intestinal mucosa [1]. Canine IBD is still a clinical exclusion diagnosis, and the therapy is mostly based on experience and includes a combination of elimination diet, antibiotics and immunosuppressive drugs and is often frustrating [2]. The pathogenesis of IBD is incompletely understood, but environmental, immunologic and genetic factors may play an important role. The presence of the intestinal microbiome as well as the loss of tolerance to commensal microorganisms is important for the development of intestinal inflammation in mouse models of IBD $[1,3,4]$. There is good evidence that excessive mucosal immune response to components of the microbiome, due to abnormal or impaired effector or regulatory cell activity, is important in the pathogenesis of human IBD in genetically predisposed individuals [4]. Several studies about human and canine IBD have indicated significant differences in the intestinal bacterial microbiota between IBD patients and healthy controls $[4,5]$. Suchodolski et al. [5] demonstrated a significant difference in the 
composition of the duodenal microflora between dogs with idiopathic IBD and healthy control dogs.

Pattern recognition receptors (PRRs) are responsible for the recognition of microbe-associated molecular pattern (MAMPs) [6,7]. Toll like receptors (TLRs) are an important group belonging to this family. To date, 13 members of the TLR family have been identified in mammals, and TLR 1-9 seem to be conserved between human and mouse $[8,9]$. These receptors recognize MAMPs present on diverse microbes including Gram-positive and Gram-negative bacteria, fungi, viruses and parasites. The TLRs recognizing bacterial MAMPs are TLR2 (lipopeptides, peptidoglycan (PGN), and lipoteichoic acid), TLR4 (lipopolysaccharide (LPS)), TLR5 (flagellin), and TLR9 (bacterial and viral unmethylated CpG oligonucleotides). TLRs are expressed by cells belonging to the innate immune system such as macrophages and dendritic cells, but also by human intestinal epithelial cells (IEC) [10]. Binding of a TLR ligand results in a downstream signaling cascade with activation of several kinases and ultimately in the activation of nuclear factor- $\kappa \mathrm{B}(\mathrm{NF}-\kappa \mathrm{B})$ and the production of proinflammatory cytokines, chemokines, costimulatory molecules and reactive oxygen and nitrogen intermediates $[6,11,12]$.

However, TLR signaling is also important for gut homeostasis and epithelial barrier function [13]. Several mechanisms are responsible to induce tolerance against commensal bacteria and to protect the host against excessive inflammation. A reduced expression of TLRs and decreased responsiveness to TLR ligands as well as induction of tolerance after repeated exposure to TLR ligands in epithelial cells may reduce inflammation [7,13]. The expression of TLRs can be extracellular on the cell surface like TLR2, 4 and 5 or intracellular like TLR3 and TLR9 $[8,9,14]$. However, in mouse colonic epithelial cells, TLR9 is expressed on the apical and basolateral cell surface [15]. It has been shown that TLR 2 and 4 are expressed constitutively on primary canine colonic epithelial cells and that the expression could be stimulated with PGN for TLR2 and LPS for TLR4 [16]. On the level of mRNA expression, TLR2, TLR 4 and TLR9 were upregulated in dogs with steroid-responsive diarrhea compared to healthy dogs $[17,18]$. TLR 4 was upregulated in German Shepherd Dogs with IBD in comparison to healthy dogs, whereas TLR 5 was downregulated [19]. No significant differences were seen in the expression of TLR2 and TLR9 mRNA between German Shepherd Dogs with IBD and control dogs.19 Furthermore, polymorphisms in the TLR4 and TLR5 gene were significantly associated with IBD in German shepherd dogs [20]. On the level of protein expression in dogs, there are no studies published up to now, but this group produced canine-specific antibodies and evaluated protocols for the use in dogs [21].

The purpose of this study was to determine the expression of TLR2, TLR4, TLR5 and TLR9 using these antibodies [21] for immunohistochemistry in dogs with chronic enteropathies (FR or SR) compared to healthy control dogs (HCD) before and after standard therapy and to test the hypothesis that there is a dysregulation of expression of TLRs on the protein level in dogs suffering of CCE.

\section{Material and Methods}

\section{Healthy control dogs}

Control biopsies were taken at necropsy from 20 healthy Beagle dogs, which served as placebo controls in unrelated pharmacologic studies. This group included 8 males and 12 females (all intact), 11-168 months old (median 87 months), with body weights from 5.4 to 15.5 $\mathrm{kg}$ (median $10 \mathrm{~kg}$ ). These dogs did not receive any drugs, were clinically healthy with no signs of diarrhea or vomiting, and showed no abnormalities in complete blood count, serum biochemical profile, and urinalysis. Furthermore, parasitic and bacterial analyses of fecal samples were performed and the samples were free of Giardia sp., Salmonella sp., Campylobacter sp. and other potential causes of diarrhea. Full thickness biopsies of mesenteric lymph nodes and at least 6 biopsies with an endoscopic biopsy forceps as well as full thickness biopsies from duodenum and colon were taken at necropsy directly after euthanasia. The biopsies were kept in $4 \%$ neutral-buffered formalin. The samples were examined by a board-certified pathologist and were histologically unremarkable [17].

\section{Dogs with chronic enteropathies}

Forty dogs with signs of chronic gastrointestinal disease referred to the Small Animal Teaching Hospital of the University of Bern between November 2006 and February 2009 were included in this prospective study. Some of the dogs described here were used in another study and information about these dogs and the study protocol is available elsewhere [17]. Selection criteria included a history of chronic diarrhea with or without vomiting that lasted for at least 3 weeks, exclusion of identifiable underlying disorders, and histopathological evidence of intestinal inflammatory cellular infiltrate. Owners of dogs signed a letter of consent in which they agreed to participate in initial and follow-up diagnostic evaluation, including endoscopic exams before and after therapy. None of the dogs had been treated with antibiotics, corticosteroids, or antacids at least 7 days before entering the study. All experimental procedures were approved by the Cantonal Committee for Animal Experimentation, Bern, Switzerland (118-05).

All dogs were given a clinical score using the canine IBD activity index (CIBDAI) as established by Jergens et al. [22] and were classified as clinically insignificant (score $0-3$ ), mild (4-5), moderate (6-8), or severe (9-18) before and after therapy. Furthermore, the dogs were classified according to their predominant clinical signs as having upper or lower gastrointestinal disease or both. Duodenoscopy and colonoscopy were performed in all dogs except those with severe hypoalbuminemia $(\mathrm{n}=7$; all $<18 \mathrm{~g} / \mathrm{L})$, where a $36 \mathrm{~h}$ fasting period required for colonoscopy was considered to be detrimental. After endoscopy, all dogs were treated initially with an elimination $\operatorname{diet}^{1}$ for 14 days. Recommendations usually state that dogs should be fed an appropriate formulation for at least 4-6 weeks [23]. The elimination diet trial in our study was accomplished for 14 days based on recently obtained evidence, that diet trials of 10 days are adequate to show complete remission in dogs with food responsive diarrhea [24].

Dogs that responded to the elimination diet in the first 14 days (clinical signs improved or resolved) were assigned to the FR group. This group included 20 dogs, 12 males (10 intact, 2 neutered) and 8 females (6 intact, 2 neutered), 6-76 months old (median 21.5 months), with body weights from 7.5 to $61 \mathrm{~kg}$ (median $27.9 \mathrm{~kg}$ ). Breeds in this group included: Bernese Mountain Dog (3), German Shepherd Dog (2), Golden Retriever (2), mixed breed (2) and one of each belonging to Whippet, West Highland White Terrier, Leonberger, Great Dane, Malinois, Labrador Retriever, Schwyzer Laufhund, Shi-Tzu, Alaskan Malamute, English Setter and Weimaraner breed.

The dogs that did not respond in the first 14 days of treatment (clinical signs persisted while on the elimination diet) were assigned to the SR group and were given oral prednisolone $(1 \mathrm{mg} / \mathrm{kg}$ PO q12 h) for 14 days followed by a tapering dosage (50\% reduction every 2 weeks if possible). This group included 20 dogs, 12 males (5 intact, 7 neutered) 
and 8 females (1 intact, 7 neutered), 32-154 months old (median 61 months), with body weights from 2.9 to $71.7 \mathrm{~kg}$ (median $20.5 \mathrm{~kg}$ ). Breeds in this group included: mixed breed (3), Dachshund (2), Shar Pei (2), Yorkshire Terrier (2) and one of each belonging to Rottweiler, Bull Mastiff, Cavalier King Charles Spaniel, Papillion, Beauceron, Bernese Mountain Dog, Pug, German Shepherd Dog, Golden Retriever, West Highland White Terrier and Malinois breed.

The protocol that was used in this study was described before [17]. Seven of 20 dogs in the SR group were clinically classified as having protein-losing enteropathy (PLE). These dogs showed a panhypoproteinemia and a severe hypoalbuminemia (all $<18 \mathrm{~g} / \mathrm{L}$ ). Because of the severe hypoalbuminemia and the need of a quick response, the elimination diet and the treatment with prednisolone in these dogs were started immediately after the first endoscopy.

\section{Tissue samples}

Dogs were prepared for endoscopy by withholding food for $36 \mathrm{~h}$ and administering a colonic lavage solution ${ }^{2}$ by gastric intubation $(2$ doses of $30 \mathrm{~mL} / \mathrm{kg}$ of body weight 6-8 h apart). At least six adequate mucosal biopsy specimens were obtained from the duodenum $(\sim 10 \mathrm{~cm}$ below the caudal duodenal flexure), and middle portion of the descending colon, or from where lesions were visible. An endoscopic score [25] was assigned based on mucosal appearance and on the severity of changes. Samples for subsequent histopathological evaluation were placed in $4 \%$ neutral-buffered formalin or paraformaldehyd for $24 \mathrm{~h}$ before embedding in paraffin. Biopsy specimens were examined by a board-certified pathologist who was blinded with regard to clinical diagnosis and treatments used.

\section{Immunohistochemistry}

The paraffin embedded tissue sections were deparaffinized and rehydrated with a xylol-alcohol series and washed three times with Tris-buffered saline (TBS) ${ }^{3}$. The epitope retrieval was performed with citrate buffer $\left(\mathrm{pH}\right.$ 6) in a standardized microwave $\left(92^{\circ} \mathrm{C}\right.$ for 15 minutes). Sections were cooled to room temperature for 20 minutes. After another washing step with TBS, Triton-X 100 (1\%) was utilized for 10 minutes to penetrate the cell membrane. To minimize background, the endogenous peroxidase was blocked with methanol and $3 \%$ hydrogen peroxide solution for 15 minutes and the samples were washed again with TBS. To reduce nonspecific binding of proteins, samples were incubated 30 minutes with $5 \%$ normal goat serum. The sections were incubated overnight at $4^{\circ} \mathrm{C}$ with the primary antibody (purified rabbit-anti canine TLR; TLR2 diluted 1:500, TLR4 diluted 1:50, TLR5 diluted 1:150 and TLR9 diluted 1:300 in $0.01 \mathrm{M}$ Tris- $\mathrm{NaCl} \mathrm{pH} \mathrm{7.4,} \mathrm{respectively)} \mathrm{[21].} \mathrm{The} \mathrm{secondary} \mathrm{antibody}{ }^{4}$ was administered for 15 minutes by room temperature. After a washing step with TBS, sections were stained with biotin-streptavidinhorseradish-peroxidase $\mathrm{s}^{5}$ for 15 minutes by room temperature and washed again. Amino-9-ethyl-carbazole was used as chromogen 6 . After counterstaining with hematoxilin, sections were cover-slipped with an aqueous mounting medium ${ }^{7}$ and digital pictures were taken and analyzed. Positive (mesenterial lymph node) and negative (intestine and mesenterial lymph node without primary antibody) controls were run with every batch of samples.

Coloration of cells as well as the staining of the epithelium was evaluated separately. The degree of expression of TLRs in the biopsy specimens was blindly evaluated by two examiners (MS and AZ) as described previously [26]. In brief, the scoring scheme graded epithelium and cells as follows: epithelium: $0=$ the same as background; $0.5=$ close to background; $1=$ well-marked positivity; $1.5=$ strong positivity; $2=$ very strong positivity. Cells: $0=$ no positive cells visible; $0.5=$ sporadic single cells; $1=$ scattered single cells; $1.5=$ scattered cells with discrete clusters; $2=$ large groups or clusters of cells; $2.5=$ dense dissemination.

\section{Statistical analysis}

All statistical analyses were performed with $\mathrm{NCSS}^{8}$ (www.ncss.com). A Kruskal-Wallis One-way ANOVA and Kruskal-Wallis MultipleComparison z-Value Test (Dunn's Test) was used to compare the three groups in regard to cells and staining of epithelium in colon and duodenum. Spearman Correlation test was used to show correlations between cell count and staining of epithelium in duodenum and colon. A repeated Measures ANOVA was used to compare the measurements within the three groups over time. For CIBDAI, a 2-way repeated measures routine with groups (FR, SR) and time (before, after) was utilized. A p-value $<0.05$ was considered significant.

\section{Results}

\section{CIBDAI}

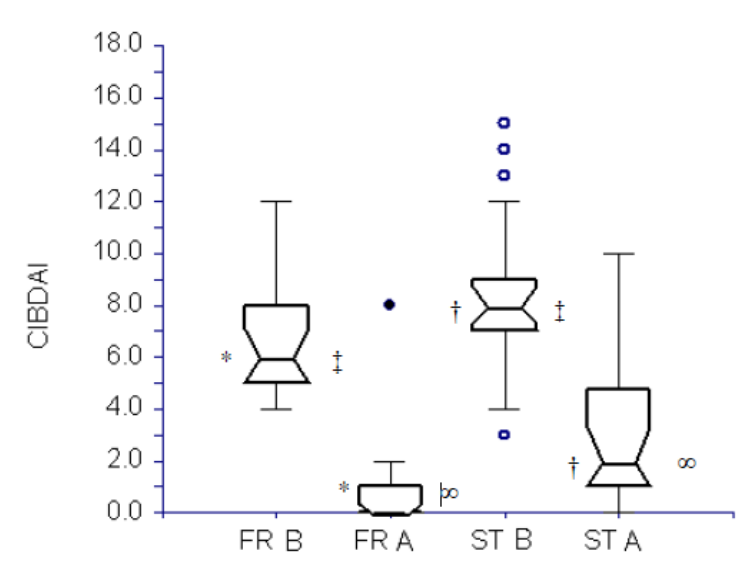

Figure 1: Canine inflammatory bowel disease activity index (CIBDAI) before (B) and after (A) therapy in 20 dogs with foodresponsive (FR) and 20 dogs with steroid responsive (SR) diarrhea. The CIBDAI was significantly higher in the SR group compared to FR before and after therapy ( $\mathrm{p}=0.022$ and 0.011 , respectively). Furthermore, there was a significant improvement over time within both groups (both $\mathrm{p}<0.001){ }^{*}, \infty$, $\neq$ and $\dagger$ show significance.

The CIBDAI was significantly higher in the SR (median 8 , range 4-15) group compared to FR (median 6, range 4-12) before and after ( SR; median 2, range $0-10$; FR median 0 , range $0-2$ ) therapy $(\mathrm{p}=0.022$ and 0.011 , respectively). As expected, there was a significant improvement over time within the FR and SR group $(\mathrm{p}<0.001)$ (Figure $1)$.

\section{TLR 2}

As a positive control, slides from the same lymph node from a healthy dog were used in all runs. The staining pattern revealed 
positive cells predominantly in the cortex (T-cell area and dendritic cells) and medulla (dendritic cells, macrophages and B-cell area).

In the duodenum, the epithelium was positive stained in the villus and in the crypt area. The staining was more intense in the apical epithelial cells and attenuated in the crypt area both in the duodenum and the colon. Miscellaneous TLR2 positive cell populations were found in the lamina propria and submucosa. Positive cells stained mainly cytoplasmatic and in some extent granular but only scattered membranous. The distribution of TLR2 positive cells in the lamina propria, likely lymphocytes, dendritic cells and possibly macrophages, was disseminated. In addition to these cell populations cells of the submucosal plexus (Meissner's plexus), possibly neurons, were also positive for TLR2. Most positive cells were found in the healthy control dogs. TLR2 positive cells were significantly down-regulated in the duodenum and the colon in FR (cell scoring range before therapy 0,5-2,5 (median 1,5) and 0,5-2 (median 1) and cell scoring range after therapy $0,5-2$ (median 1,5$)$ and $0-2$ (median 1$)$, respectively) and SR (cell scoring range before therapy 0,5-2 (median 1,5) and 0,5-1,5 (median 1) and cell scoring range after therapy 0,5-2 (median 1) and 0,5-1,5 (median 1), respectively) group compared to HCD (cell scoring range 1,5-2,5 (median 2,5) and 1,5-2,5 (median 2)) before and after therapy (all $\mathrm{p}<0.0001)$. In regard to the epithelium, the expression of TLR2 in the duodenum was higher in FR before therapy ( $p=0.009$, cell scoring range before therapy $0-2$ (median 1)) compared to HCD (cell scoring range 0-1 (median 0,5)). No significance was shown in expression of TLR2 positive epithelium in FR group compared to HCD after therapy as well as SR group before and after therapy compared to HCD.

\section{TLR 4}

Expression of TLR4 positive cells in the mesenteric lymph node from a healthy dog, which served as positive control, was accentuated in the germinal centre (B- and T-lymphocytes, dendritic cells). The staining pattern was considerably membranous (Figure 2 ).

Intestinal epithelial cells were negative, as no expression of TLR4 was detected neither in the epithelium of the duodenum nor the colon in healthy and diseased dogs. However, in the lamina propria of the duodenum and the colon invading cells stained positive for TLR4. The staining pattern of positive cells was particularly membranous and infrequently granular-cytoplasmatic. Beside lymphocytes endothelial cells of intestinal capillaries stained positive. TLR4 positive cells were up-regulated in duodenum in FR before $(\mathrm{p}=0.008$; cell scoring range $0,5-2,5$ (median 1,5$)$ ) and after therapy $(\mathrm{p}=0.02$; cell scoring range 0,5-2,5 (median1,5)) compared to HCD (cell scoring range 0-2 (median 0,75$)$ ). In the colon, TLR4 positive cells were significantly upregulated in FR (cell scoring range before therapy 0,5-2 (median 1,5) and after therapy 0,5-2 (median 1)) and SR (cell scoring range before therapy 0,5-2 (median 1) and after therapy 0-2,5 (median 1,5) group before $(\mathrm{p}=0.0009)$ and after therapy $(\mathrm{p}=0.0002)$ compared to HCD (cell scoring range $0-1,5$ (median 0,5 )

\section{TLR 5}

The staining pattern of the mesenteric lymph node (positive control) revealed positive lymphocytes and macrophages in the perivascular sinus as well as lymphocytes in the parafollicular space (Tcell area) and the border area of the lymph follicle (B-cell area) as shown in Figure 2.
In the duodenum, the epithelium was stained continuously in both the villus and crypt area. The staining in the villus was accentuated in the apical epithelial cell domain. Furthermore, an apical accentuated coloration was found in epithelial cells in the colon. In the duodenum and colon, different cell populations were positive for TLR5 with a disseminated distribution. There was a basal granular staining of intraepithelial cells in the crypts, supposedly lymphocytes. The number of these cells decreased from duodenum to colon. In the lamina propria, lymphocytes, macrophages and endothelial cells stained positive. The staining pattern seemed to be granular and more cytoplasmatic than membranous. In full thickness biopsies of healthy dogs, the apical epithelium was more intensely stained than the crypts. In addition, the Meissner's plexus was positively staining for TLR5. In addition to the positive lymphocytes in lymph follicles in full thickness biopsies of the duodenum, also dendritic cells in the middle of lymph follicles were positively stained. However, cells were not precisely defined with other surface markers.

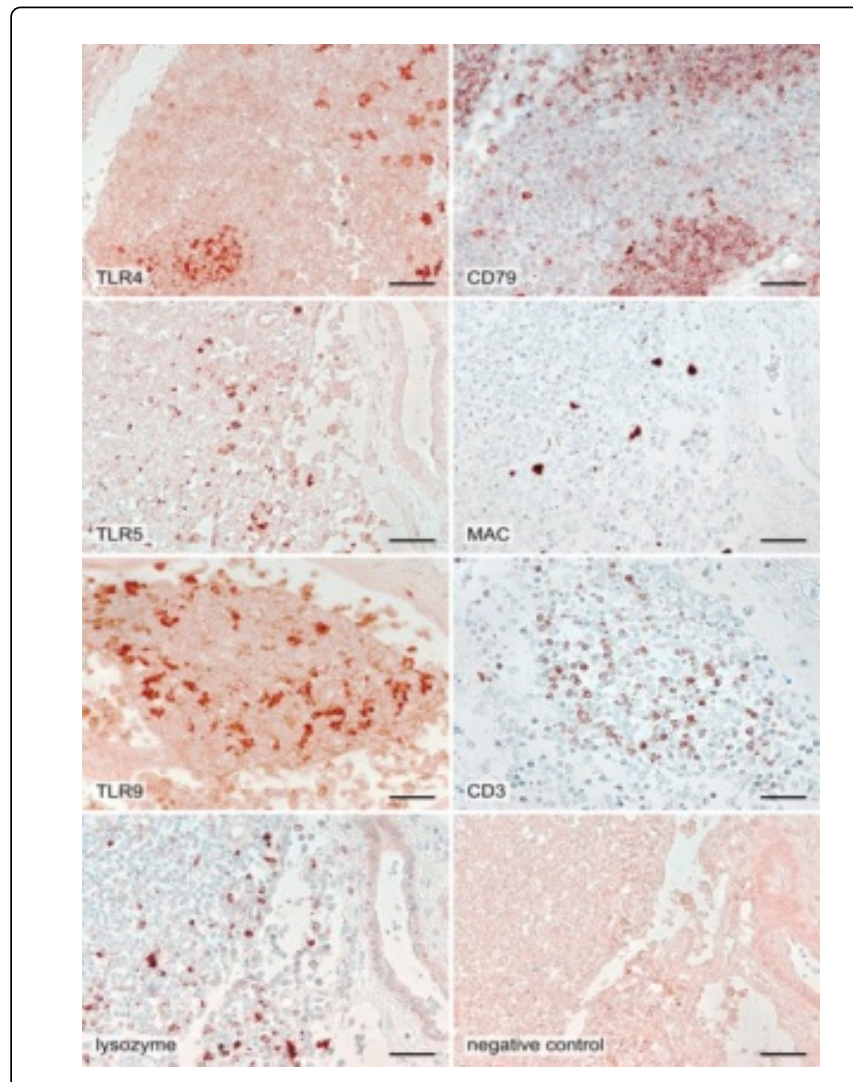

Figure 2: Representative immunohistochemistry results of TLR4, TLR5 and TLR9 antibodies compared with CD79, MAC and CD3 staining at a comparable site of a canine reactive mesenterial lymph node. Lysozyme staining and a negative control section are added for comparison Bar $=100 \mu \mathrm{m}$. 


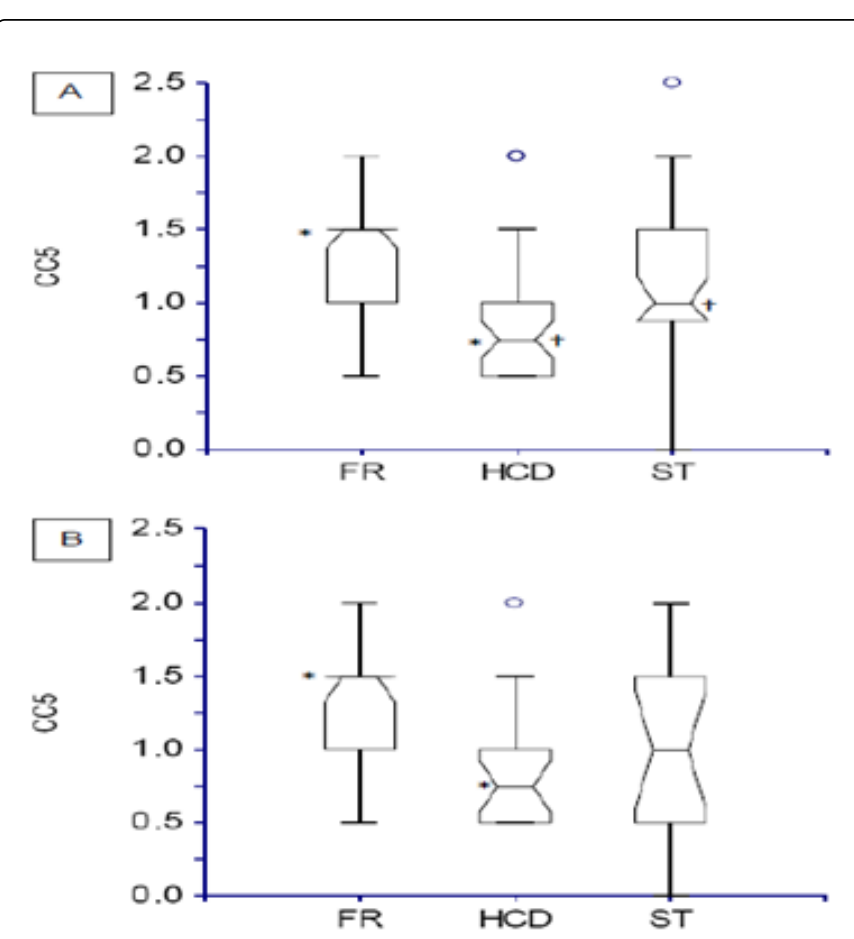

Figure 3: TLR5-positive cells in the colon (CC5) in dogs with food responsive (FR) diarrhea, healthy control dogs (HCD) and steroid responsive (SR) diarrhea before (A) and after therapy (B) The FRD and SR groups expressed significantly more TLR5 positive cells in the colon before therapy compared to the HCD ( $p=0.005)$. After therapy, only the FR group still yielded significantly more positive cells in the colon $(\mathrm{p}=0.020)$.

The FR and SR groups expressed significantly more TLR 5 positive cells in the colon ( $\mathrm{p}=0.005$, cell scoring range $0,5-2,5$; median 1,5 and 1 , respectively), but not the duodenum before therapy compared to the HCD (cell scoring range 0,5-1,5; median 0,75; Figure 3A). After therapy, only the FR group still yielded significantly more positive cells in the colon ( $\mathrm{p}=0.020$; cell scoring range $0,5-2$; median 1,5 ; Figure $3 \mathrm{~B}$ ). In the epithelium, there was no significant difference in staining patterns between the groups neither in the duodenum nor in the colon before and after therapy.

The expression of TLR5 in cells and epithelium correlated before and after therapy in duodenum $(\mathrm{r}=0.506, \mathrm{p}<0.001$; and $\mathrm{r}=0.584$, $\mathrm{p}<0.001$, respectively) and colon $(\mathrm{r}=0.501, \mathrm{p}<0.001$; and $\mathrm{r}=0.513$, $\mathrm{p}<0.001$, respectively). Therefore, a positive correlation exists between the staining of the cells from the lamina propria mucosae and the cells of the epithelium mucosae in FR and SR.

\section{TLR 9}

The same lymph node as used for TLR2, 4 and 5 was also taken as a positive control for TLR9 in all runs. TLR9 positive cells were accentuated in the lymph follicles (B-cell area), but also some lymphocytes in the precortex and cortex were positively stained (Figure 2). The cellular staining was granular and cytoplasmatic.

Most positive cells were found in healthy dogs, and in decreasing tendency in dogs suffering from FR, SR or PLE. The positive cells in
HCD were equally distributed in the crypts and the villi. In general, there were more positive cells in the duodenum, but staining of the epithelium was stronger in the colon than in the duodenum. Cells positive for TLR9 were composed of a homologous group of small cells, supposedly lymphocytes. Meissner's plexus yielded also positive staining.

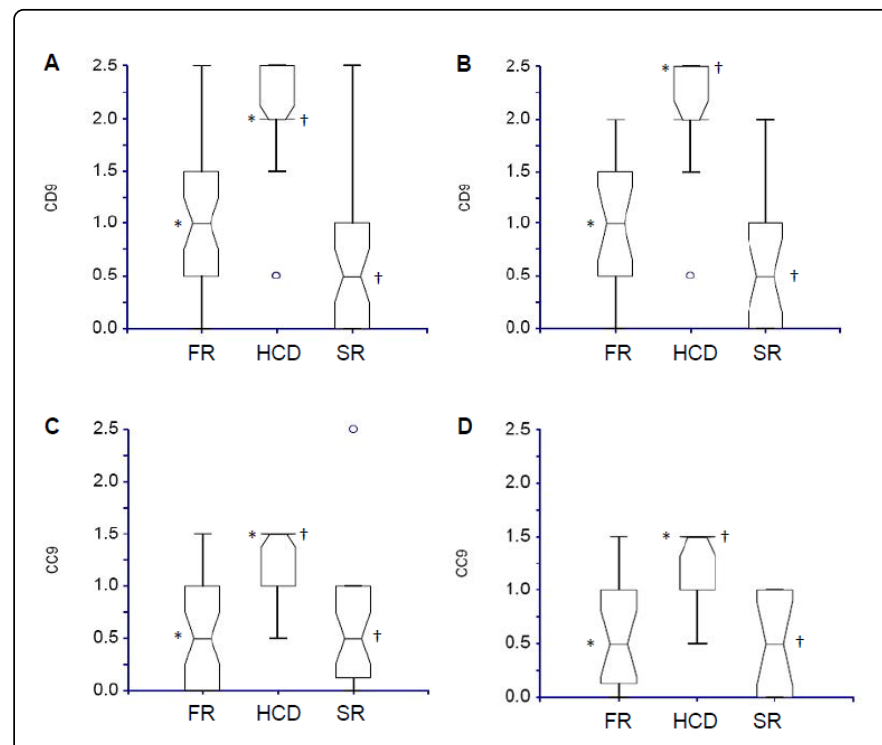

Figure 4: TLR9-positive cells in the duodenum (CD9) and colon (CC9) in dogs with food responsive (FR) diarrhea, healthy control dogs (HCD) and steroid responsive (SR) diarrhea before (A, C) and after therapy (B, D). The expression of TLR9 positive cells in duodenum and colon was significantly lower in the diseased dogs compared to HCD before and after therapy (all $\mathrm{p}<0.001$ ).

Expression of TLR9 positive cells in duodenum and colon was significantly lower in FR (cell scoring range before therapy 0-2,5 (median 1) and 0-1,5 (median 0,5) and cell scoring range after therapy $0-2$ (median 1) and 0-1,5 (median 0,5), respectively) and SR group (cell scoring range before therapy $0-2,5$ (median 0,5$)$ and $0-1$ (median 0,5 ) and cell scoring range after therapy $0-2$ (median 0,5 ) and $0-1$ (median 0,5 ), respectively) than in HCD (cell scoring range duodenum $0,5-2,5$ (median 2) and colon 0,5-1,5 (median 1,5) before and after therapy (all $\mathrm{p}<0.001$, Figure 4$)$. The epithelium was stained significantly weaker in the FR group in duodenum $(\mathrm{p}=0.009)$ and colon $(\mathrm{p}=0.036)$ before therapy (epithelium scoring range $0-1$ (median 0 ) and $0-1$ (median 0,5)) compared to HCD (epithelium scoring range 0-1 (median 0,75) and $0-1,5$ (median 1)) (Figures $5 \mathrm{~A}$ and $\mathrm{C}$ ). After therapy, the epithelial staining was significantly weaker in FR (epithelium scoring range duodenum 0-1 (median 0,25) and colon 0-1,5 (median 0,5) and SR group (epithelium scoring range duodenum $0-1$ (median 0 ) and colon $0-1$ (median 0,5$)$ for both duodenum $(\mathrm{p}=0.023)$ and colon $(\mathrm{p}=0.013)$ (Figures 5B and 5D).

The expression of TLR9 correlated within cells or epithelium in the 2 segments before (cells $\mathrm{r}=0.611, \mathrm{p}<0.001$; epithelium $\mathrm{r}=0.519$, $\mathrm{p}<0.001$ ) and after therapy (cells $\mathrm{r}=0.649, \mathrm{p}<0.001$; epithelium $\mathrm{r}=0.667$, $\mathrm{p}<0.001$ ). As there is a positive correlation between the staining of the cells of the lamina propria mucosae and the cells of the epithelium mucosae a strong staining of cells is attended by a strong staining of the epithelium. 
The epithelium was stained significantly weaker in the FR group in duodenum ( $\mathrm{p}=0.009)$ and colon $(\mathrm{p}=0.036)$ before therapy (epithelium scoring range $0-1$ (median 0 ) and $0-1$ (median 0,5) compared to HCD (epithelium scoring range $0-1$ (median 0,75 ) and $0-1,5$ (median 1) (Figures 5A and 5C). After therapy, the epithelial staining was significantly weaker in FR (epithelium scoring range duodenum $0-1$ (median 0,25) and colon 0-1,5 (median 0,5) and SR group (epithelium scoring range duodenum $0-1$ (median 0$)$ and colon $0-1$ (median 0,5$)$ for both duodenum $(p=0.023)$ and colon $(p=0.013)$ (Figure 5B, D). The expression of TLR9 correlated within cells or epithelium in the 2 segments before (cells $\mathrm{r}=0.611, \mathrm{p}<0.001$; epithelium $\mathrm{r}=0.519, \mathrm{p}<0.001$ ) and after therapy (cells $\mathrm{r}=0.649, \mathrm{p}<0.001$; epithelium $\mathrm{r}=0.667$, $\mathrm{p}<0.001$ ). As there is a positive correlation between the staining of the cells of the lamina propria mucosae and the cells of the epithelium mucosae a strong staining of cells is attended by a strong staining of the epithelium.
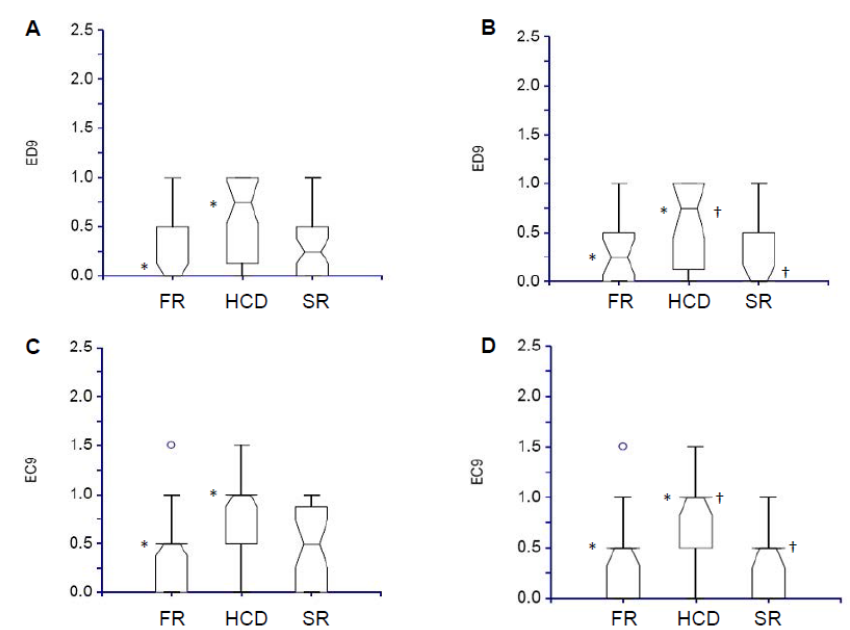

Figure 5: TLR9-positive epithelium in the duodenum (ED9) and colon (EC9) in dogs with food responsive (FR) diarrhea, healthy control dogs (HCD) and steroid responsive (SR) diarrhea before (A, C) and after therapy (B, D) the epithelium was stained significantly weaker in the FR group in duodenum and colon before therapy (A, C) compared to $\mathrm{HCD}$ ( $\mathrm{p}=0.009$ and 0.036 , respectively). After therapy, the epithelium was stained significantly weaker in FR and SR group compared to HCD for both duodenum $(\mathrm{p}=0.023)$ and colon $(\mathrm{p}=0.013)$.

\section{Discussion}

Pattern recognition receptors like TLRs are responsible for the recognition of MAMPs [6,7] present on diverse microbes including Gram-positive and Gram-negative bacteria, fungi, viruses and parasites. Binding of a TLR ligand results in a downstream signaling cascade with activation of several kinases and ultimately in the activation of NF- $\mathrm{KB}$ and the production of proinflammatory cytokines, chemokines, costimulatory molecules and reactive oxygen and nitrogen intermediates $[6,11,12]$. To the author's knowledge, this is the first study evaluating protein expression of TLR2, TLR4, TLR5 and TLR9 in the canine intestine. Furthermore, we could prove our hypothesis that there is a dysregulation in TLR expression on the protein level in dogs suffering of CCE.
TLR2 expression was significantly higher in the intestinal epithelium of FR before therapy compared to HCD, but not in SR. Furthermore, a significant down-regulation of TLR2 positive invading cells in duodenum and colon was found in FR and SR before and after therapy. These results are partially discrepant to findings from previously performed studies in dogs $[17,18]$ and men $[26,27]$ which showed an up-regulation of TLR2 mRNA expression in IBD. After mRNA expression, several regulatory mechanisms are involved like post-transcriptional and translational modifications and protein degradation, which could regulate and modify the abundance of protein expression [28,29]. Furthermore, a few studies in men provided evidence of up-regulation of TLR2 in individuals suffering from IBD, not only on the level of mRNA but also protein expression $[26,27,30]$. In regard to the bacterial population, there are some alterations of the microbiome towards a more gram negative flora and less diversity in canine IBD [5,31]. A reduced TLR2 expression as a result of diminished gram positive bacterial population may be a reason for TLR2 downregulation in dogs with CE, as TLR2 expression is influenced by LTA and PGN, two principal components of the gram positive bacterial wall [10]. An enhanced expression of TLR2 (mRNA and protein) was demonstrated by Voltan and coworkers in colonic mucosa and IEC of mice after either supplementation or incubation of Lactobacillus crispatus M247 [32]. In dogs, stimulation of duodenal biopsies with TLR ligands revealed anti-inflammatory effects, whereas the impact of Enterococcus faecium was limited [33]. Furthermore, TLR2 appears to mediate some host-protective qualities in mice by restoring intestinal epithelial barrier integrity and reducing colonic mucosal inflammation $[34,35]$. Last but not least, regulatory $\mathrm{T}$ cells (Tregs) play a crucial role in modulating immune reaction, in particular maintaining tolerance to self-antigen. In duodenum of dogs suffering from IBD, diminished numbers of Tregs were found compared to healthy controls [36]. In vitro and in vivo studies in mice revealed regulatory mechanisms of TLR2 signaling in T cell (effector and regulatory T cell) homeostasis $[37,38]$. On one hand TLR2 ligands enhance the proliferation and IL-2 production of effector T cells while transiently suppressing Treg function, which is regained with declining quantity of TLR2 ligands. On the other hand, TLR2 agonists increase Treg numbers. This mechanism could enable the host to combat pathogens or inducing tolerance against commensals and controlling inflammation accordingly. Diminished TLR2 expression in dogs with $\mathrm{CE}$ may therefore point toward a defective T cell homeostasis. Another theory for our findings may be the downregulation of TLR2 as a consequence of severe inflammation in order to protect the host of overwhelming inflammatory response.

In contrast to TLR2 and TLR9, TLR4 expression was significantly up-regulated in invading cells in the duodenum in FR and in the colon in FR and SR group before and after therapy. A trend towards an increased duodenal expression of TLR4 positive cells in SR group before and after therapy was recorded, though significance was not reached. Hence, a larger study population may be needed to achieve statistical significance. Similar findings were found on mRNA $[17,19]$ and protein level in canine and human IBD, respectively [39]. Igarashi and colleagues indicated an upregulation of mRNA of TLR2, 4 and 9 in miniature dachshunds with inflammatory colorectal polyps compared to healthy control dogs [40]. In addition, enhanced transcriptional and protein level of TLR4 positive intestinal LP macrophages were reported in human IBD [30]. Pronounced TLR4 expression of invading cells of diseased dogs may suggest increased inflammation and added contact to LPS, possible through translocated bacteria in response to disturbed intestinal permeability. There are conflicting results in regard to TLR4 
protein expression in intestinal epithelial cells [30,39]. We did not detect any TLR4 positive IEC, neither in dogs suffering from CE nor in controls. In agreement to our findings, human IEC remained negative for TLR4 protein expression in IBD and control samples [30] Furthermore, our findings indicate altered TLR4 expression in CCE compared to healthy controls. Dysregulation of TLR4 may therefore play a role in the pathogenesis of canine IBD as already indicated in German Shepherd Dogs [20].

Similar to findings of TLR4, dogs suffering from CCE showed a significant upregulation of TLR5 positive cells in the lamina propria mucosae (lymphocytes, dendritic cells and endothelial cells) in the colon. Furthermore, TLR5 expression correlated in cells and epithelium within the intestinal segment in duodenum and colon. These results point towards a dysregulated expression and may indicate a pro-inflammatory function of TLR5 in SR and FRD. After therapy, only the FR group exhibited an upregulated expression of TLR5. Since the SR group was treated with prednisolone, it is possible that the gastrointestinal inflammation in these dogs was suppressed through suppression of proinflammatory transcription factors (for example NFкВ) [41]. Furthermore, Amsterdam and coworkers pointed out that steroid hormones can induce apoptosis in hematopoietic cells like monocytes, macrophages and T-lymphocytes which are involved in inflammatory processes [42]. In accordance with the literature, our study suggests a potential influence of glucocorticoids on the expression of TLR5 by induction of apoptosis in cells belonging to the innate immune system, but further studies are needed to investigate this relationship. In Jewish people, the heterozygous carriage of TLR5stop, a dominant-negative TLR5 polymorphism, is negatively associated with Crohn's disease [43]. In these people, TLR5-stop is unable to activate NF- $\kappa \mathrm{B}$ response to flagellin. However, mice with complete loss of TLR5 function show a dysregulation of the bacterial gut flora such as an increased tendency to develop severe gastrointestinal inflammation [44]. A canine genetic study revealed one different DNA sequence variation (SNP) in the TLR5 gene significantly associated with IBD in German Shepard Dogs [20]. Furthermore, they spotted two SNPs in the TLR5 gene to be significantly protective for IBD. A recently published in vitro and ex vivo study from the same group indicated a hyper-responsiveness to flagellin in German shepherd dogs carrying the TLR5 risk-associated haplotype for canine IBD [45]. Consequently, TLR5 may have proinflammatory, but as well some homeostatic qualities.

Unlike TLR5, TLR9 expression was significantly decreased in cells and epithelium of the duodenum and colon of dogs suffering from SR and FR compared to HCD. The epithelium stained significantly weaker for TLR9 in the FR group before therapy, and in FR and SR group after therapy compared to HCD. An upregulation of TLR9 on the surface can lead to inflammation or tolerance in mice. [15] Rose et al. [46] showed an increased sensitivity to DSS (dextran sulfate sodium) induced intestinal damage and reduced ability to repair intestinal injury in TLR9 deficient mice leading to the suggestion that TLR9 plays a key role in homeostatic processes in the intestine. Our group showed a significantly higher expression of mRNA for TLR9 in duodenum and colon in SR dogs, [17] but no significant difference before and after therapy. A recent study revealed as well a higher expression of TLR9 on mRNA and protein level in rats with TNBS (2,4,6-trinitrobenzensulfonic acid) induced colitis compared to controls with severity of colitis correlating with TLR9 expression [47]. Similar to our results, mRNA levels of TLR9 were lower in dogs with FR compared to healthy control dogs [33]. However, another study found no difference in expression of mRNA for TLR9 in GSD with IBD and healthy controls [19]. The divergence of these findings could be explained by the variety of mechanisms of regulation after expression of mRNA. Another explanation could be that the biopsies were taken in a non-inflamed or only mildly inflamed area. The expression of mRNA as well as protein levels of TLR2 and TLR4 were significantly increased in the inflamed colonic mucosa of children with IBD compared to controls, whereas no differences in expression of mRNA and protein was recognized in biopsies of non-inflamed mucosa [27].

One limitation of this study is that the healthy control group consisted of Beagle dogs only. It has been shown in several studies that Beagles may not represent a good control group as they may have subclinical GI disease despite being clinically healthy. However, other studies using healthy greyhounds as controls have found similar mRNA expression levels of TLRs in the duodenum than found in healthy Beagles [19]. Another limitation of the study is the missing double staining of TLR-positive cells to better define the cells staining positive for TLR2, TLR4, TLR5 or TLR9. Possible alteration in the cell population would be possible to detect before and after therapy. Furthermore, it may be interesting to state which cells express TLR2, 4, 5 and 9 before and after therapy, as this may give some hints which kind of cytokine profile is presented and possible may be further investigated. Uematsu and coworkers [48] found a high expression of TLR 5 mRNA in CD11c+ lamina propria cells in the intestine of mice. In addition, they elucidated a flagellin induced stimulation of proinflammatory cytokines like IL-6 and IL-12 through CD11c+ intestinal lamina propria cells in TLR5+/+ but not in TLR5-/- knock out mice [48]. Their data suggested that CD11c+ lamina propria cells induce an innate immune response via TLR 5 by detecting pathogenic flagellated bacteria. However, Kathrani et al. [49] revealed a significant decrease of CD11c+ cells (a marker of human and murine dendritic cells) in the duodenum, ileum and colon of dogs with inflammatory bowel disease compared to healthy control dogs [49].

In conclusion, this is the first study evaluating protein expression of TLR2, TLR4, TLR5 and TLR9 in the canine intestine. TLR4 and TLR5 expression measured by immunohistochemistry was found to be upregulated in inflammatory cells of the lamina propria in the duodenum of FR (TLR4) and colon in dogs with FR and SR (TLR4 and TLR5). However, TLR2 and TLR9 expression was downregulated in lamina propria cells and in the epithelium (TLR9) in dogs with FR and SR. The downregulation of TLR9 in cells and epithelium seen in CE compared to healthy dogs points towards the important role that TLR9 also plays in gut homeostasis in mice and men. Possible new therapeutic approaches to impair TLR4 and TLR5 expression or induce TLR2 and TLR9 activation with corresponding ligands (e.g, synthetic lipopeptides or synthetic CpGs, respectively) [38] in dogs with chronic enteropathies may then become worthwhile.

${ }^{1}$ Purina LA salmon and rice or Biomill fish and rice, Biomill SA, Granges-Marnand, Switzerland

${ }^{2}$ Each liter contains $60 \mathrm{~g}$ polyethylene glycol (PEG) 4000, $1.46 \mathrm{~g}$ sodium chloride, $0.745 \mathrm{~g}$ potassium chloride, $1.68 \mathrm{~g}$ sodium bicarbonate and $5.68 \mathrm{~g}$ sodium sulfate

${ }^{3}$ Tris-buffered saline; $60 \mathrm{~g}$ Trishydroxymethylaminomethan, $4 \mathrm{~g} \mathrm{KCl}$, $160 \mathrm{~g} \mathrm{NaCl}$, dilution 1:10 with Aqua dest, $\mathrm{pH} 7.4$

${ }^{4}$ Biotylinatd mouse anti rabbit IgG, DAKO Kit K5003, DAKO Schweiz AG, Baar, Switzerland

${ }^{5}$ Biotin-streptavidin-horseradish-peroxidase, DAKO Kit K5003, DAKO Schweiz AG, Baar, Switzerland 


\author{
${ }^{6}$ Amino-9-ethyl-carbazole (AEC), DAKO Kit K5003, DAKO \\ Schweiz AG, Baar, Switzerland \\ ${ }^{7}$ Aquatex ${ }^{\oplus}$ Merck Art. Nr. $1.0856250 \mathrm{ml}$ for microscopy, Darmstadt, \\ Germany
}

${ }^{8}$ Number Cruncher Statistical Systems (NCSS), Version May 2008, Kaysville, Utah, USA

\section{References}

1. German AJ, Hall EJ, Day MJ (2003) Chronic Intestinal Inflammation and Intestinal Disease in Dogs. J Vet Intern Med 17: 8-20.

2. Craven M, Simpson JW, Ridyard AE, Chandler ML (2004) Canine inflammatory bowel disease: retrospective analysis of diagnosis and outcome in 80 cases (1995-2002). J Small Anim Pract 45: 336-342.

3. Allenspach K (2011) Clinical Immunology and Immunopathology of the Canine and Feline Intestine. Vet Clin North Am Small Anim Pract 41: 345-360.

4. Strober W (2006) Immunology unraveling gut inflammation. Science 313:1052-1054.

5. Suchodolski JS, Xenoulis PG, Paddock CG, Steiner JM, Jergens AE (2010) Molecular analysis of the bacterial microbiota in duodenal biopsies from dogs with idiopathic inflammatory bowel disease. Vet Microbiol 142: 394-400.

6. Akira S, Uematsu S, Takeuchi O (2006) Pathogen Recognition and Innate Immunity. Cell 124: 783-801.

7. Michelsen KS, Arditi M (2007) Toll-like receptors and innate immunity in gut homeostasis and pathology. Curr Opin Hematol 14: 48-54.

8. Takeda K, Akira S (2005) Toll-like receptors in innate immunity. Int Immunol 17: 1-14.

9. Bowie A, O'Neill LA (2000) The interleukin-1 receptor/Toll-like receptor superfamily: signal generators for pro-inflammatory interleukins and microbial products. J Leukoc Biol 67: 508-514.

10. Cario E, Brown D, McKee M, Devaney LK, Gerken G, et al. (2002) Commensal-associated molecular patterns induce selective toll-like receptor-trafficking from apical membrane to cytoplasmic compartments in polarized intestinal epithelium. Am J Pathol 160: 165-73.

11. Akira S, Takeda K (2004) Toll-like receptor signalling. Nat Rew Immunol 4: 499-511.

12. O'Neil LA (2006) Toll-like receptors signal: what we know and what we don't know. Curr Opin Immunol 18: 3-9.

13. Rakoff-Nahoum S, Paglino J, Eslami-Varzaneh F, Edberg S, Medzhitov R (2004) Recognition of Commensal Microflora by Toll-Like Receptors is Required for Intestinal Homeostasis. Cell 118: 229-241.

14. Burgener IA, Jungi TW (2008) Antibodies specific for human or murine Toll-like receptors detect canine leukocytes by flow cytometry. Vet Immunol Immunopathol 124: 184-191.

15. Lee J, Mo J, Katakura K, Alkalay I, Rucker AN (2006) Maintenance of colonic homeostasis by distinctive apical TLR 9 signalling in intestinal epithelial cells. Nat Cell Biol 8: 1327-1336.

16. Swerdlow MP, Kennedy DR, Kennedy JS, Washabauet JR, Henthorn SP, et al. (2006) Expression and function of TLR2, TLR4 and Nod2 in primary canine colonic epithelial cells. Vet Immunol Immunopathol 114: 313-319.

17. Burgener IA, Konig A, Allenspach K, Sauter SN, Boisclair J, et al. (2008) Upregulation of Toll-like Receptors in Chronic Enteropathies in Dogs. J Vet Intern Med 22: 553-560.

18. McMahon LA, House AK, Catchpole B, Elson-Riggins J, Riddle A, et al. (2010) Expression of Toll-like receptors 2 in duodenal biopsies from dogs with inflammatory bowel disease is associated with severity of disease. Vet Immunol Immunopathol 135: 158-163.

19. Allenspach K, House A, Smith K, McNeill FM, Hendricks A, et al. (2010) Evaluation of mucosal bacteria and histopathology, clinical disease activity and expression of Toll-like receptors in German shepherd dogs with chronic enteropathies. Vet Microbiol 146: 326-335.
20. Kathrani A, House A, Catchpole B, Murphy A, German A, et al. (2010) Polymorphisms in the TLR4 and TLR5 gene are significantly associated with inflammatory bowel disease in German Shepherd Dogs. PLoS ONE 5: e15740.

21. Huber S, Roosje PJ, Janda J, Schnyder M, Jungi TW, et al. (2011) Characterization of antibodies specific for canine TLR4, 5 and 9 by ELISA, western blotting and immunohistochemistry. Vet Immunol Immunopathol 144: 247-254.

22. Jergens AE, Schreiner CA, Frank DE, Niyo Y, Ahrens FE, et al. (2003) A scoring index for disease activity in canine inflammatory bowel disease. J Vet Intern Med 17: 291-297.

23. Roudebush P (2005) Adverse reactions to foods: Allergies versus intolerance. In: Ettinger SJ, Feldman EC, eds. Textbook of Veterinary Internal Medicine, 6th ed. Philadelphia, PA: WB Saunders 566-570.

24. Allenspach K, Wieland B, Grone A, Gaschen F (2007) Chronic enteropathies in dogs evaluation of risk factors for negative outcome. J Vet Intern Med 21: 700-708.

25. Jergens AE, Moore FM, Haynes JS, Miles KG (1992) Idiopathic inflammatory bowel disease in dogs and cats: 84 cases (1987-1990). J Am Vet Med Assoc 201:1603-1608.

26. Frolova L, Drastich P, Rossmann P, Klimesova K, Hogenova HT (2008) Expression of Toll-like receptor 2 (TLR2), TLR4, and CD14 in biopsy samples of patients with inflammatory bowel diseases: upregulated expression of TLR2 in terminal ileum of patients with ulcerative colitis. J Histochem Cytochem 56: 267-274.

27. Szebeni B, Veres G, Dezsofi A, Rusai K, Vannay A, et al. (2008) Increased expression of Toll-like receptor (TLR) 2 and TLR4 in the colonic mucosa of children with inflammatory bowel disease. Clin Exp Immunol 151: 34-41.

28. Vogel C, Marcotte EM (2012) Insights into the regulation of protein abundance from proteomic and transcriptomic analyses. Nat Rev Genet 13: 227-232.

29. Anderson L, Seilhamer JA (1997) Comparison of selected mRNA and protein abundances in human liver. Electrophoresis 18: 533-537.

30. Hausmann M, Kiessling S, Mestermann S, Webb G, Spottl T, et al. (2002) Toll-like receptors 2 and 4 are up-regulated during intestinal inflammation. Gastroenterol $122: 1987-2000$.

31. Suchodolski JS, Dowd SE, Wilke V, Steiner JM, Jergens AE (2012)16S rRNA gene pyrosequencing reveals bacterial dysbiosis in the duodenum of dogs with idiopathic inflammatory bowel disease. PLoS One 7: e39333.

32. Voltan S, Castagliuolo I, Elli M, Longo S, Brun P, et al.(2007) Aggregating phenotype in Lactobacillus crispatus determines intestinal colonization and TLR2 and TLR4 modulation in murine colonic mucosa. Clin Vacc Immunol 14: 1138-1148.

33. Schmitz S, Henrich M, Neiger R, Werling D, Allenspach K (2014) Stimulation of duodenal biopsies and whole blood from dogs with foodresponsive chronic enteropathy and healthy dogs with Toll-like receptor ligands and probiotic Enterococcus faecium. Scand J Immunol 80: 85-94.

34. Cario E, Gerken G, Podolsky DK (2004) Toll-like receptor 2 enhances ZO-1-associated intestinal epithelial barrier integrity via protein kinase C. Gastroenterol 127: 224-238.

35. Cario E, Gerken G, Podolsky DK (2007) Toll-like receptor 2 controls mucosal inflammation by regulating epithelial barrier function. Gastroenterol 132: 1359-1374.

36. Junginger J, Schwittlick U, Lemensieck F, Nolte I, Trautwein MH (2012) Immunohistochemical investigation of Foxp3 expression in the intestine in healthy and diseased dogs. Vet Res 43: 23.

37. Liu H, Komai-Koma M, Xu D, Liew YF (2006) Toll-like receptor 2 signaling modulates the functions of $\mathrm{CD} 4+\mathrm{CD} 25+$ regulatory $\mathrm{T}$ cells. Proc Natl Acad Sci 103: 7048-7053.

38. Sutmuller RP, den Brok MH, Kramer M, Bennink JE, Toonen WJL, et al. (2006) Toll-like receptor 2 controls expansion and function of regulatory T cells. J Clin Invest 116: 485-494. 
Citation: Schnyder M, Oevermann A, Doherr MG, Luckschander N, Burgener IA, et al. (2018) Dysregulation of Toll-Like Receptors in Dogs with Chronic Enteropathies. J Inflamm Bowel Dis Disor 3: 127. doi:10.4172/2476-1958.1000127

Page 9 of 9

39. Cario E, Podolsky DK (2000) Differential alteration in intestinal epithelia cell expression of Toll-like receptor 3 (TLR3) and TLR4 in inflammatory bowel disease. Infect Immun 68: 7010-7017.

40. Igarashi H, Ohno K, Maeda S, Kanemoto H, Fukushima K, et al. (2014) Expression profiling of pattern recognition receptors and selected cytokines in miniature dachshunds with inflammatory colorectal polyps. Vet Immunol Immunopathol 159: 1-10.

41. De Bosscher K, Vanden Berghe W, Haegeman G (2003) The interplay between the glucocorticoid receptor and nuclear factor-kappaB or activator protein-1: molecular mechanisms for gene repression. Endocr Rev 24: 488-522.

42. Amsterdam A, Sasson R(2002)The anti-inflammatory action of glucocorticoids is mediated by cell type specific regulation of apoptosis. Mol Cell Endocrinol 189: 1-9.

43. Gewirtz AT, Vijay Kumar M, Brant SR, Duerr RH, Nicolae DL, et al. (2006) Dominant-negative TLR5 polymorphism reduces adaptive immune response to flagellin and negatively associates with Crohn's disease. Am J Physiol Gastrointest Liver Physiol 290: G1157-1163.
44. Vijay Kumar M, Sanders CJ, Taylor RT, Kumar A, Aitken JD, et al. (2007) Deletion of TLR5 results in spontaneous colitis in mice. J Clin Invest 117: 3909-3921.

45. Kathrani A, Holder A, Catchpole B, Alvarez L, Simpson K, et al. (2012) TLR5 Risk-Associated Haplotype for Canine Inflammatory Bowel Disease Confers Hyper-Responsiveness to Flagellin. PLOS ONE 7: e30117.

46. Rose WA, Sakamoto K, Leifer SA (2012) TLR9 is important for protection against intestinal damage and for intestinal repair. Sci Rep 2: 574.

47. Zhang han Dai, Bei Tan, Hong Yang, Ou Wang,Jia-ming Qian, et al. (2015) 1,25-hydroxyvitamin D relieves colitis in rats via down-regulation of toll-like receptor 9 expression. Croat Med J 56: 515-524.

48. Uematsu S, Jang MH, Chevrier N, Guo Z, Kumagai Y,et al. (2006) Detection of pathogenetic intestinal bacteria by Toll-like-receptor 5 on intestinal CD11c+ lamina propria cells. Nat Immunol 7: 868-874.

49. Kathrani A, Schmitz S, Priestnall SL (2011) CD 11+ cells are significantly decreased in the duodenum, ileum and colon of dogs with inflammatory bowel disease. J Comp Pathol 145: 359-366. 